\title{
Editorial:
}

\section{Towards a multidimensional scientific approach to improve clinical practices for infertility treatment}

\author{
Yu-hang ZHU ${ }^{1}$, Fan QU $U^{\dagger \$ 1,2}$ \\ ${ }^{\prime}$ Women's Hospital, Zhejiang University School of Medicine, \\ Hangzhou 310006, China \\ ${ }^{2}$ Institute for Women's Health, University College London, London \\ NW3 2PF, the United Kingdom \\ ${ }^{\dagger}$ E-mail: syqufan@zju.edu.cn
}

https://doi.org/10.1631/jzus.B1801014

Polycystic ovary syndrome (PCOS) is the most common metabolic and endocrine disorder in women, leading to infertility. However, there is no general agreement concerning how to diagnose and treat PCOS. The Rotterdam consensus statement from the European Society of Human Reproduction and Embryology/American Society for Reproductive Medicine, the Chinese diagnostic criteria and consensus statement, and the clinical practice guideline from the Endocrine Society in the USA are widely recognized. Guidance has been provided for clinical practice based on a comparative analysis of the above three practice guidelines or consensus statements (Wang et al., 2018a). High body mass index (BMI) has no negative effect on the outcome of in vitro fertilization (IVF) in Chinese patients with PCOS; however, the conclusion may be limited by the retrospective design and potential bias (Pan et al., 2018). Neonatal birth weight is positively affected by both maternal pre-pregnancy body mass index (pre-BMI) and gestational weight gain (Du et al., 2017). Normal body weight is very important for conception. Women with PCOS are almost 3 times more likely to be obese than those without PCOS; however, no

\footnotetext{
${ }^{\ddagger}$ Corresponding author

(D) ORCID: Fan QU, https://orcid.org/0000-0003-1851-1514

(c) Zhejiang University and Springer-Verlag GmbH Germany, part of Springer Nature 2018
}

specific interventions are available to induce weight loss, and drugs are used to treat other symptoms of the syndrome or obesity in the general population. A network meta-analysis found that the amount of weight loss differed significantly according to the choice of drugs (in descending order): liraglutide, orlistat, and metformin. Liraglutide alone, liraglutide/ metformin, and metformin alone significantly reduced waist circumference, but no change was found with orlistat, indicating liraglutide appears superior to the other drugs in reducing weight and waist circumference (Wang et al., 2018b). IVF, as a choice for more than 1000000 infertile couples each year, gives rise to the birth of over 3000000 babies worldwide.

Acupuncture, a traditional medical practice that originated in China, may be a valuable addition to healthcare (Robinson, 2017). Traditional Chinese medicine (TCM) is widely used by women to improve health, from preventing miscarriage in early pregnancy to alleviating menopausal symptoms. In recent years, many infertile women with PCOS have chosen TCM as an adjunct when they undergo IVF-embryo transfer. Increasing evidence has demonstrated that acupuncture-like therapies, including electroacupuncture (EA) and transcutaneous electrical acupoint stimulation (TEAS), are effective in treating gynecological disorders, especially infertility. Acupuncture appears to significantly improve the recovery of the menstrual cycle and decreases the BMI and luteinizing hormone (LH) level in women with PCOS $(\mathrm{Qu}$ et al., 2016). TEAS using a frequency of $2 / 100 \mathrm{~Hz}$ could help to improve IVF outcomes partly by increasing neuropeptide $\mathrm{Y}$ levels in follicular fluid $(\mathrm{Qu}$ et al., 2017a). A group consensus has been achieved on the principles of clinical application of EA/TEAS in treating various kinds of reproductive disorders, including PCOS, pain induced by oocyte retrieval, diminished ovarian reserve, embryo transfer, and 
oligospermia/asthenospermia (Qu et al., 2017b). The effects of TCM therapy on IVF outcomes depend on many factors, such as the TCM diagnosis, the timing of TCM treatment, selection of herbs/acupoints, expertise and qualification of the practitioner, types of acupuncture (traditional needling acupuncture, EA, laser acupuncture, or auricular acupuncture), and processing and formulation of herbs. Although some findings do not support the use of acupuncture to improve the rate of live births among women undergoing IVF (Smith et al., 2018), standardized guidelines for better clinical trial design of acupuncture for PCOS have been proposed (Wu et al., 2016).

With continuous advances in science and technology as well as social progress, medicine is moving toward a bio-psychological-social model. Women are more influenced by social psychology than men in terms of social division of labor, the position of family life, and the influence of traditional culture. Infertility involves both husband and wife, but disruption of the menstrual cycle caused by intense emotional fluctuations and psychological setbacks leads to a series of physiological and neurotransmitter alterations in women. Clinically, we should not only consider the disease itself, but also physiology and psychology as a whole. With a third of the burden of disease for women aged 15-44 years resulting from poor sexual and reproductive health, these issues may have a psychosocial component, with stress and anxiety playing a role (Robinson, 2017). Women treated for infertility have deeper depression and anxiety, which increases over time, and differences in emotional distress are based on pregnancy status (Vahratian et al., 2011). In a retrospective analysis of 145456 pregnant women, use of antidepressants, specifically selective serotonin reuptake inhibitors, during gestation increased the risk of autism spectrum disorder in children (Boukhris et al., 2016). Therefore, effective psychological intervention for pregnant women is particularly important. A meta-analysis suggested that psychosocial interventions for couples in treatment for infertility, in particular cognitivebehavioral therapy, could be efficacious, both in reducing psychological distress and in improving clinical pregnancy rates (Frederiksen et al., 2015). TEAS using a frequency of $2 / 100 \mathrm{~Hz}$ may also relieve anxiety in infertile women (Xing et al., 2018). Perhaps modern acupuncture therapy combined with psycho- logical intervention will provide new clinical approaches to the treatment of infertility.

To standardize clinical trials, the following items may be useful for the design of further research on the effects of TCM on IVF outcomes. (1) Selection of herbs and/or acupoints should be based on the correct TCM diagnosis. (2) Standardized acupuncture methods or processing and formulation of herbs should be applied to enhance comparability among different studies. (3) Live birth should be the primary outcome. (4) Data on hormones and psychological indices during treatment should be collected. (5) As international differences in infertile patient experiences, expectations, and knowledge of TCM may lead to variable IVF outcomes, cooperation is urgently needed to better understand the effects of TCM treatment. (6) To resolve the confusion caused by different protocols of TCM treatment during IVF and intracytoplasmic sperm injection (ICSI) in various centers, an international multicenter study group should be established to evaluate the effects of acupuncture on IVF and ICSI outcomes. (7) Confounders that have been shown to influence IVF outcomes should be taken into account, including lifestyle factors (maternal and paternal age, weight, vitamin and iodine intake, alcohol and caffeine consumption, smoking, substance misuse, stress, environmental pollutants, oxidative stress, and other such factors), gynecological factors (duration and cause of infertility, presence and characteristics of uterine fibroids, and other such factors), number of previous IVF or ICSI cycles, presence of male factors, and race and ethnicity. (8) For studies using acupuncture, Standards for Reporting Interventions in Clinical Trials of Acupuncture criteria should be applied to make protocols reproducible. (9) Detailed, fixed protocols based on the correct diagnosis according to TCM theories and expert opinion should be established. (10) A sufficient dose of treatment over the most appropriate time period should be provided. (11) Appropriate psychological intervention should be provided when necessary. (12) As there is increasing evidence that children conceived with assisted reproductive technology (ART) are at higher risk of poor perinatal outcomes, birth defects, and epigenetic disorders, and since the underlying mechanisms have not been identified, continuous follow-up of children after ART and TCM treatment 
is of great importance as they progress through adolescence into adulthood (Lu et al., 2013).

\section{Overview}

Infertility has increasingly become an important social issue. TCM and psychological intervention have certain advantages in the treatment of infertility, even in the improvement of offspring health. More standardized, international multi-center clinical trials are still needed to strengthen the evidence-based basis for effective treatment.

\section{Conflict of interest}

Yu-hang ZHU and Fan QU declare that they have no conflict of interest.

\section{References}

Boukhris T, Sheehy O, Mottron L, et al., 2016. Antidepressant use during pregnancy and the risk of autism spectrum disorder in children. JAMA Pediatr, 170(2):117-124. https://doi.org/10.1001/jamapediatrics.2015.3356

Du MK, Ge LY, Zhou ML, et al., 2017. Effects of prepregnancy body mass index and gestational weight gain on neonatal birth weight. J Zhejiang Univ-Sci B (Biomed \& Biotechnol), 18(3):263-271. https://doi.org/10.1631/jzus.B1600204

Frederiksen Y, Farver-Vestergaard I, Skovgård NG, et al., 2015. Efficacy of psychosocial interventions for psychological and pregnancy outcomes in infertile women and men: a systematic review and meta-analysis. $B M J$ Open, 5(1):e006592. https://doi.org/10.1136/bmjopen-2014-006592

Lu YH, Wang N, Jin F, et al., 2013. Long-term follow-up of children conceived through assisted reproductive technology. J Zhejiang Univ-Sci B (Biomed \& Biotechnol), 14(5):359-371. https://doi.org/10.1631/jzus.B1200348

Pan XM, Lin Z, Li N, et al., 2018. Effects of body mass index on the outcomes of in vitro fertilization in Chinese patients with polycystic ovary syndrome: a retrospective cohort study. J Zhejiang Univ-Sci B (Biomed \& Biotechnol), 19(6):490-496. https://doi.org/10.1631/jzus.B1800113

Qu F, Wu Y, Hu XY, et al., 2016. The effects of acupuncture on polycystic ovary syndrome: a systematic review and meta-analysis. Eur J Integr Med, 8(1):12-18. https://doi.org/10.1016/j.eujim.2016.02.001

Qu F, Wang FF, Wu Y, et al., 2017a. Transcutaneous electrical acupoint stimulation improves the outcomes of in vitro fertilization: a prospective, randomized and controlled study. Explore, 13(5):306-312. https://doi.org/10.1016/j.explore.2017.06.004

Qu F, Li R, Sun W, et al., 2017b. Use of electroacupuncture and transcutaneous electrical acupoint stimulation in reproductive medicine: a group consensus. $J$ Zhejiang Univ-Sci B (Biomed \& Biotechnol), 18(3):186-193. https://doi.org/10.1631/jzus.B1600437

Robinson N, 2017. Integrating acupuncture: are there positive health outcomes for women? J Zhejiang Univ-Sci B (Biomed \& Biotechnol), 18(3):233-238. https://doi.org/10.1631/jzus.B1600260

Smith CA, de Lacey S, Chapman M, et al., 2018. Effect of acupuncture vs sham acupuncture on live births among women undergoing in vitro fertilization: a randomized clinical trial. JAMA, 319(19):1990-1998. https://doi.org/10.1001/jama.2018.5336

Vahratian A, Smith YR, Dorman M, et al., 2011. Longitudinal depressive symptoms and state anxiety among women using assisted reproductive technology. Fertil Steril, 95(3):1192-1194. https://doi.org/10.1016/j.fertnstert.2010.09.063

Wang FF, Pan JX, Wu Y, et al., 2018a. American, European, and Chinese practice guidelines or consensuses of polycystic ovary syndrome: a comparative analysis. J Zhejiang Univ-Sci B (Biomed \& Biotechnol), 19(5):354-363. https://doi.org/10.1631/jzus.B1700074

Wang FF, Wu Y, Zhu YH, et al., 2018b. Pharmacologic therapy to induce weight loss in women who have obesity/ overweight with polycystic ovary syndrome: a systematic review and network meta-analysis. Obes Rev, 19(10): 1424-1445. https://doi.org/10.1111/obr.12720

Wu Y, Robinson N, Hardiman PJ, et al., 2016. Acupuncture for treating polycystic ovary syndrome: guidance for future randomized controlled trials. $J$ Zhejiang Univ-Sci B (Biomed \& Biotechnol), 17(3):169-180. https://doi.org/10.1631/jzus.B1500301

Xing LF, Wu Y, He YJ, et al., 2018. Transcutaneous electrical acupoint stimulation alleviates the anxiety levels of IVF: a prospective, randomized and controlled study. Eur $J$ Integr Med, 20:126-130. https://doi.org/10.1016/j.eujim.2018.05.003

\section{中文概要}

题 目: 通过多维度的科学方法提高不孕症治疗的临床实 践水平

概 要: 不孕症正日渐成为一个重要的社会关切问题。中 医及心理干预在不孕症的治疗, 甚至提高子代健 康水平等方面具有一定优势, 但仍需更加规范及 国际性的多中心临床研究来加强其有效临床治 疗的循证基础。

关键词: 多维度; 不孕症; 临床实践 